\title{
Topological Realizations of Calkin Algebras on Frechet Domains of Unbounded Operator Algebras
}

\author{
K. SCHMZ̈̈DGEN
}

$D$ sei ein dichter linearer Teilraum eines separablen Hilbertraumes und $\mathscr{L}+(\mathscr{D})$ die maximale Op*-Algebra auf $D$, versehen mit der gleichmäßigen Topologie $\tau_{\mathscr{D}} \cdot$ Wir setzen voraus, da $\mathcal{D}$ bezüglich der Graphtopologie von $\mathscr{L}+(\mathscr{D})$ ein Frechetraum ist. Weiter sei $\mathscr{C}(\mathscr{D})$ das zweiseitige - -Ideal aller Operatoren aus $\mathscr{L}+(\mathscr{D})$, die beschränkte Teilmengen von $\mathscr{D}$ in relativ kompakte Teilmengen abbilden. Es wird untersucht, wann die Faktoralgebra $\mathcal{A}(\mathcal{D}):=\mathscr{L}+(\mathcal{D}) / \mathscr{C}(\mathcal{D})$, versehen mit der Faktorraumtopologie, eine topologische Realisierung als $\mathrm{Op} *$-Algehra besitzt.

IІусть $\mathscr{D}$ плотное линейное подпространство сепарабельного гильбертова пространства и пусть $\mathscr{L}+(\mathscr{D})$ максимальная Ор -алгебра над $\mathcal{D}$, сиаб̈ённая равномерной топфлогие $\tau_{\mathrm{D}}$. ПІредіолагается, что $\mathcal{D}$ явлнется пространством Фреше относительн топологии порождённой граф-нормами операторов из $\mathscr{L}+(D)$. Через $\mathscr{C}(\mathcal{D})$ обозначается двусторонний *-иде́л тех операторов из $\mathscr{L}+(\mathcal{D})$, которые переводят ограниченные подмножества пространства $D$ в относительно компактные понмнонества. Исследуется вопрос о том, юогда факторалгебра $\mathcal{A}(\mathscr{D}):=\mathscr{L}+(\mathscr{D}) / \mathscr{C}(\mathscr{D})$ снабжённая топологией факторпространства допускает топологическую реализацию как Ор*-алгебра.

Let $D$ be a densc linear subspace of a separable Hilbert space and let' $\mathscr{L}+(D)$ be the maximal Op*algebra on $D$ endowed with the uniform topology $\tau \mathscr{D}$. Suppose $\mathcal{D}$ is a Frechet space with respect to the graph topology of $\mathscr{L}+(D)$. Let $\mathscr{C}(D)$ denote the two-sided *-ideal of all operators in $\mathscr{L}+(D)$ which map bounded subsets of $\mathcal{D}$ into relatively compact subsets. We study the question of when the quotient algebra $\mathcal{A}(\mathscr{D}):=\mathscr{L}+(\mathscr{D}) / \mathscr{C}(\mathscr{D})$, endowed with the quotient topology, has a topological realization as an Op*algebra.

\section{Introduction}

Let $\mathscr{D}$ be a dense linear subspace of a separable complex Hilbert space $\mathscr{H}$ endowed with the graph topology $t$ (see Section 1 for precise definitions). Suppose $D[t]$ is a Frechet space. Let $\mathscr{C}(\mathscr{D})$ denote the set of all operators in $\mathscr{L}^{+}(\mathscr{D})$ which map each bounded subset of $\mathscr{D}[t]$ into a relatively compact subset of $\mathscr{D}[l]$. Then $\mathscr{C}(\mathscr{D})$ is a $\tau_{D^{D}}$-closed two-sided $*$-ideal of $\mathscr{L}^{+}(\mathscr{D})$ which contains the finite rank operators in $\mathscr{L}^{+}(\mathscr{D})$ as a dense subset $[15,7]$. (Note that in [15] the ideal $\mathscr{C}(\mathscr{D})$ is denoted by $V_{0}(t, l)$.) The quotient algebra"A(D) $:=\mathscr{L}^{+}(\mathscr{D}) / \mathscr{C}(\mathscr{D})$ is called the Calkin algebra on the domain $\mathscr{D}$. Let $\hat{\imath}$ denote the quotient topology on $\mathscr{A}(\mathscr{D})$ of $\mathscr{L}^{+}(\mathcal{D})\left[\tau_{\mathscr{D}}\right]$. Obviously, $\mathcal{A}(\mathcal{D})[\hat{\imath}]$ is a topological $*$-algebra. If $\mathscr{D}=\mathscr{H}$, then $\mathcal{A}(\mathscr{D})=\dot{A}(\mathscr{H})$ is the usual Calkin algebra on the Hilbert space $\mathscr{H}$. It should be mentioned that if $\mathscr{D}[t]$ is a Montel space, then $\mathscr{C}(\mathcal{D})=\mathscr{L}^{+}(\mathcal{D})$ and hence the Calkin algebra $\mathcal{A}(\mathscr{D})$ is trivial.

In his classical paper [3] CALKIN constructed a class of faithful isometric *-representations of the $\mathrm{C}^{*}$-algebra $\mathcal{A}(\mathscr{H})$ (see [11] for a modern treatment). In this paper we investigate the corresponding problem for the Calkin algebra $\mathcal{A}(\mathscr{D})$ on the Frechet domain $\mathscr{D}[t]$ : Does there exist a faithful *-representation $\pi$ of $\mathcal{A}(\mathscr{D})$ which is a homeomorphism of $\dot{A}(\mathcal{D})[\hat{\tau}]$ onto $\pi(\mathcal{A}(\mathscr{D}))\left[\tau_{\mathscr{D}(x)}\right]$ ? For the domain $l_{2} \otimes d, d$ the space of all finite complex sequences, this problem has been considered in [9]. Note that $l_{2} \otimes d[t]$ is not a Frechet space: 
Let us briefly describe our main results concerning the above question:

Given'a free ultrafilter $\mathcal{U}$ on $\mathrm{N}$, we define in Section 2 a *-representation $\pi u$ of $\mathcal{A}(\mathscr{D})$ in a similar way as. in the case $\mathscr{D}=\mathscr{H}$. We show that $\pi u$ is faithful and that $\pi_{u^{-1}}$ is continuous (Theorem 2.1). Let $\tau_{n}$ denote the finest locally convex topology on $\mathscr{L}^{+}(\mathcal{D})$ for which the positive cone $\mathscr{L}^{+}(\mathscr{D})_{+}$is normal [12]. If $\tau_{n}=\tau_{\mathscr{D}}$ on $\mathscr{L}^{+}(\mathscr{D})$, then each *-representation $\pi u$ is continuous and hence a homeomorphism (Theorem 2.2).

In Section 3 we obtain a converse of the latter in some sense. Suppose that the graph topology $t$ on $\dot{D}$ is generated by a sequence of strongly commuting self-adjoint operators whose restrictions to $\mathscr{D}$ are in $\mathscr{L}^{+}(\mathscr{D})$. Under this additional assumption we prove that if $\tau_{n} \neq \tau_{\mathscr{D}}$ on $\mathscr{L}^{+}(\mathscr{D})$, then there is no continuous faithful *-representation of $\mathcal{A}(\mathcal{D})[\hat{\boldsymbol{\tau}}]$ (Theorem 3.1).

\section{Preliminaries}

In this section we collect some definitions and notations (see e.g. $[8,10]$ ) needed later and we prove-some preliminary lemmas.

1.1 Let $\mathcal{D}$ be a 'dense linear subspace of a complex Hilbert space $\mathscr{H}$ and let $\mathscr{L}^{+}(\mathscr{D})$ $:=\left\{a \in\right.$ End $\mathscr{D}: \mathscr{D} \subseteq \mathscr{D}\left(a^{*}\right)$ and $\left.a^{*} \mathscr{D} \subseteq \mathscr{D}\right\} . \mathscr{L}^{+}(\mathscr{D})$ is a *-algebra endowed with the involution $a \rightarrow a^{+}:=a^{*} \mid \mathcal{D}$. An $O p *$ :algebra $\mathscr{B}$ on $\mathscr{D}$ is a $*$-subalgebra of $\mathscr{L}^{+}(\mathscr{D})$. In ' what follows we assume that $\mathscr{B}$ is an Op*-algebra on $\mathscr{D}$. Define $\mathscr{D}(\mathscr{B})=\cap\{\mathscr{D}(\bar{b})$ $: b \in \mathscr{A}\}$, where $\bar{b}$ is the closure of the operator $b$ : The graph topology $t_{\mathscr{B}}$ is the locally convex topology on $\mathcal{D}$ defined by the seminorms $\|\varphi\|_{b}:=\|b \varphi\|+\|\varphi\|, b \in \mathscr{B}$. In case $\mathscr{B}=\mathscr{L}^{+}(\mathscr{D})$ we simply write $t$ for $t_{\mathscr{A}}$.

Let $\left(\varphi_{n}: n \in \mathrm{N}\right)$ be a sequence of vectors $\varphi_{n} \in \mathscr{H}$ and let $\varphi \in \mathscr{H}$. Suppose $\mathcal{U}$ is a filter on $\mathrm{N}$. We write $\varphi=\mathrm{w} \cdot \lim \varphi_{n}$ if $\lim _{n}\left\langle\varphi_{n}, \psi\right\rangle=\langle\varphi, \psi\rangle$ for all $\psi \in \mathscr{H}$ and $\varphi$ $=\mathrm{w}-\lim _{\mathcal{H}} \varphi_{n}$ if $\lim u\left\langle\varphi_{n}, \psi\right\rangle=\langle\varphi, \psi\rangle$ for all $\psi \in \mathscr{H}$.

Lem m 1.1: Suppose $U$ is an ultrafilter on $\mathrm{N}$. Let $\left(\varphi_{n}: n \in \mathcal{N}\right)$ be a bounded sequence of vectors of $\mathscr{D}\left[i_{\mathscr{B}}\right]$. Let $\varphi:=\mathrm{w} \cdot \lim _{u} \varphi_{n}$.

(i) Then, $\varphi \in \mathscr{D}(\mathscr{B})$ and $\bar{b} \varphi=\mathrm{w}-\lim _{\boldsymbol{u}} b \varphi_{n}$. In particular, if $0=\mathrm{w}-\lim _{\boldsymbol{u}} \varphi_{n}$, then $0^{\prime}=\mathrm{w}-\lim _{u} b \varphi_{n}$ for each $b \in \mathscr{B}$.

(ii) If $\lim _{u}\left\|\varphi_{n}\right\|=0$, then $\lim _{u}\left\|b \varphi_{n}\right\|=0$ for each $\dot{b} \in \mathscr{B}$.

(iii) If $\varphi=0$ and if the set $\left\{\varphi_{n}\right\}$ is relatively compact in $D\left[t_{\mathscr{B}}\right]$, then $\lim _{u}\left\|b \varphi_{n}^{-}\right\|=0$, for éach $b \in \mathscr{B}$.

Proof: (i) Suppose $b \in \mathscr{B}$. Since the set $\left\{b \varphi_{n}\right\}$ is bounded in the Hilbert space norm and $\mathcal{U}$ is an ultrafilter, $\lim u\left\langle b \varphi_{n}, \cdot\right\rangle$ is a continuous linear functional on $\mathscr{H}$. Hence there is a $\varphi_{0} \in \mathscr{H}$ such that $\varphi_{0^{\prime}}=\mathrm{w}$ - $\lim _{\mathcal{U}} b \varphi_{n}$. For $\psi \in \mathscr{D}\left(b^{*}\right)$, this gives

$$
\left\langle\varphi_{b}, \psi\right\rangle=\lim _{u}\left\langle b \varphi_{n} ; \psi\right\rangle=\lim _{u}\left\langle\varphi_{n}, b^{*} \psi\right\rangle=\left\langle\varphi, b^{*} \psi\right\rangle
$$

Therefore, $\varphi \in \mathscr{D}\left(b^{* *}\right) \equiv \mathscr{D}(\bar{b})$ and $\varphi_{b}=b^{* *} \varphi \equiv \cdot \bar{b} \varphi$. Since $b \in \mathscr{B}$ is arbitrary, $\varphi \in \cap\{\mathcal{D}(\vec{b}): b \in \mathscr{B}\} \equiv \mathscr{D}(\mathscr{B})$.

(ii) Since $\left\{\varphi_{n}\right\}$ is $t_{\mathscr{B}}$-bounded, $C_{b}:=\sup \left\{\left\|b^{+} b \varphi_{n}\right\|: n \in \mathbb{N}\right\}<\infty$ for $b \in \mathscr{B}$. Now the assertion follows from

$$
\left(\lim _{u}\left\|b \varphi_{n}\right\|\right)^{2}=\lim _{u}\left\langle b^{+} b \varphi_{n}, \varphi_{n}\right\rangle \leqq C_{b}\left(\lim _{\mathcal{L}}\left\|\varphi_{n}\right\|\right)=0 .
$$

(iii) Let $b \in \mathscr{B}$. Since $\left\{\varphi_{n}\right\}$ is relatively compact in $\mathscr{D}\left[t_{\mathscr{B}}\right]$, the set $\left\{b \varphi_{n}\right\}$ is relatively compact in $\mathscr{H}$. Given $\varepsilon>0$, there is a finite rank projection $F_{\varepsilon}$ on $\mathscr{H}$ such that $\left\|\left(I-F_{\varepsilon}\right) b \varphi_{n}\right\| \leqq \varepsilon$ for $n \in N$. Since $0=w-\lim _{u} b \varphi_{n}$ because of (i) and hence $\lim _{u}\left\|F_{\varepsilon} b \varphi_{n}\right\|=0$, we have $\lim _{u}\left\|b \varphi_{n}\right\| \leqq \lim _{u}\left\|\left(I-F_{\varepsilon}\right) b \varphi_{n}\right\| \leqq \varepsilon$, thus $\lim _{u}\left\|b \varphi_{n}\right\|=0$ a

The following corollary is of some interest in itself: 
Corollary 1.2: Suppose $\varphi \in \mathscr{H}$. If there is a bounded sequence $\left(\varphi_{n}: n \in \mathbb{N}\right)$ in $\mathcal{D}\left[t_{\mathscr{B}}\right]$ such that $\varphi=\mathrm{w}$ - $\lim \varphi_{n}$, then $\varphi \in \underline{D}(\mathscr{B})$.

Proof: Take an ultrafilter $\mathcal{U}$ on $N$ which contains all sets $\{n \in N: n \geqq k\}, k \in N$. Then $\varphi=w_{-} \lim _{u} \varphi_{n}$ and Lemma 1.1 (i) applies

1.2 Next we briefly discuss the topologization of the Op*-algebra $\mathscr{B}$ on $\mathscr{D}$. Let $\mathscr{B}_{h}$ $:=\left\{b \in \mathscr{B}: b=b^{+}\right\}$. Suppose $b_{1}, b_{2} \in \mathscr{B}_{h}$. We write $b_{1} \geqq b_{2}$ if $\left\langle b_{1} \varphi, \varphi\right\rangle \geqq\left\langle b_{2} \varphi, \varphi\right\rangle$ for all . $\varphi \in \mathcal{D}$. Define $\mathscr{B}_{+}:=\{b \in \mathscr{B}: b \geqq 0\}$ and $\left[b_{1}, b_{2}\right]:=\left\{b \in \mathscr{B}_{h}: b_{1} \leqq b \leqq b_{2}\right\}$. The uniform topology $\tau_{D}$ is the locally convex topology on $\mathscr{B}$ defined by the seminorms

$$
p_{\mathfrak{M}}(x):=\sup \{|\langle x \varphi, \psi\rangle|: \varphi, \psi \in \mathfrak{M}\}, \mathfrak{M} \mathcal{D} \subset \mathcal{D}\left[t_{\mathscr{B}}\right] \text { bounded. }
$$

It has been introduced in:[8]. We denote by $\tau_{n}$ the finest locally convex-topology on $\mathscr{B}$ for which the positive cone $\mathscr{B}_{+}$is normal. (All notions and facts concerning ordered vector spaces we need can be found in [12].) Since $\mathscr{B}_{+}$is $\tau_{\mathscr{D}}$-normal [13], we have$\tau_{\mathscr{D}} \subseteq \tau_{n}$. Let $\tau_{0}$ denote the finest locally convex topology on $\mathscr{B}$ for which every order interval $\left[b_{1}, b_{2}\right] ; b_{1}, b_{2} \in \mathscr{B}_{h}$, is bounded. Since $\mathscr{B}_{+}$is $\tau_{n}$-normal, all order intervals are $\tau_{n}$-bounded [12: p. 216] and hence $\tau_{n} \subseteq \tau_{0}$. In [1] the topology $\tau_{0}$ is called the $\varrho$-topo$\log y \cdot$

1.3 Let $A$ be $a *$-algebra with unit élement denoted by 1 . By a *-representation of $A$ on $\mathscr{D}$ we mean $a-*$-homomorphism $\pi$ of $A$ into $\mathscr{L}^{+}(\mathscr{D})$ satisfying $\pi(1)=I$, where $I$ is the identity map of $D$. We then write $\mathscr{D}(\pi)$ for $\mathscr{D}$ and $t_{z}$ for the graph topology of the Op*-algebra $\pi(A)$ on $\mathscr{D}(\pi)$. Suppose $\pi$ is a $*$-representation of a topological $*$-algebra $A[\tau] . \pi$ is called weakly continuous if for each $\varphi \in \mathcal{D}(\pi)$ the linear functional $\langle\pi(\cdot) \varphi, \varphi\rangle$ is continuous on $A[\tau]$. If $\pi$ is a continuous mapping of $A[\tau]$ onto $\pi(A)\left[\tau_{D_{(}(\pi)}\right]$ we say $\pi$ is continuous.

As above, let $\mathscr{B}$ be an 0 p*-algebra on $\mathscr{D}$. Let $\pi$ be a *-representation of $\mathscr{B}$ on $\mathscr{D}(\pi)$. We say $\pi$ is positive if $\pi\left(\mathscr{B}_{+}\right) \subseteq \pi(\mathscr{B})_{+}$, i.e., if $b \in \mathscr{B}$ and $b \geqq 0$ on $\mathcal{D}$ always implies that $\pi(b) \geqq 0$ on $\mathcal{D}(\pi)$. A linear functional $f$ on $\mathscr{B}$ is called positive if $f(b) \geqq 0$ for all $b \in \mathscr{B}_{+}$.

Lemma 1.3: Each positive *-representation $\pi$ of the $O p *$-algebra $\mathscr{B}$ is a continuous mapping of $\mathscr{B}\left[\tau_{n}\right]$ onto $\pi(\mathscr{B})\left[\tau_{\mathscr{D}(\pi)}\right]$.

Proof: By the polarization formula it is easy to see [13] that the uniform topology $\tau_{\mathscr{D}(x)}$ on $\pi(\mathscr{B})$ is generated by the family of seminorms

$$
p_{\mathfrak{M}}^{\prime}(\pi(x)):=\sup \{|\langle\pi(x) \varphi, \varphi\rangle|: \dot{\varphi} \in \mathfrak{M}\}, \mathfrak{M} \mathcal{M} \subset \mathcal{D}(\pi) \cdot\left[\hat{t}_{\pi}\right] \text { bounded. }
$$

Fix the bounded set $\mathfrak{M}$. Since the set $\left\{x \in \mathscr{B}: p_{\mathfrak{M}}^{\prime}(\pi(x)) \leqq 1\right\}$ is absolutely convex and $\mathscr{B}_{+}$-saturated, it is a $\tau_{n}$-neighborhood of zero in $\mathscr{B}$. This proves the continuity of $\pi$

Lemma 1.4: Suppose that $\mathcal{D}[t]$ is a Frechet space.' Let $\pi^{\prime}$ be a weakly continuous *-representation of $\mathscr{L}^{+}(\dot{D})[\tau \mathscr{D}]$. Then:

(i) $\pi$ is positive.

(ii) If $x \in \mathscr{L}^{+}(\mathcal{D})$ is bounded, then $\pi(\dot{x})$ is bounded on $\mathscr{D}(\pi)$ and $\|\pi(x)\| \leqq\|x\|$.

(iii) Suppose $x_{n} \in \mathscr{L}^{+}(D)$ for $n \in \mathrm{N}$. If $\left\{\|\cdot\| \|_{x_{n}}: \dot{n} \in \mathrm{N}\right\}$ is a generating family for the graph topology ton $\mathcal{D}$, then $\left\{\|\cdot\|_{\pi\left(x_{n}\right)}: n \in N\right\}$ is a generating family of seminorms for the graph topology $t_{\pi}$ on $\mathscr{D}(\pi)$.

Proof: (i) Suppose $x \in \mathscr{L}^{+}(\mathscr{D})_{+}$and $\varphi \in \mathscr{D}(\pi)$. By [6: Theorem 6.1] there is a net $\left\{q_{j}\right\}$ of orthogonal projections $q_{j} \in \mathscr{L}^{+}(\mathcal{D})$ (that is, $q_{j}=q_{j}^{+}$, and $q_{j}=q_{j}{ }^{2}$ ) such that $q_{j} \mathscr{H} \subseteq D$ for all $j$ and $x=\tau_{D}-\lim q_{j} x q_{j}$. Let $x_{j}$ denote the operator $q_{j} x q_{j}$ on the Hilbert 
space $q_{j} \mathscr{H}$. Since $x \in \mathscr{L}^{+}(\mathscr{D}), x_{j}$ is closed and hence bounded. Let $y_{j}$ denote the positive square root of the bounded self-adjoint operator $x_{j}$ on the Hilbert space $q_{j} \mathscr{H}$. Then $y_{j} q_{j} \in \mathscr{L}^{+}(\mathscr{D})$ and

$$
\left\|\pi\left(y_{j} q_{j}\right) \varphi\right\|^{2}=\left\langle\pi\left(q_{j} y_{j}{ }^{2} q_{j}\right) \varphi, \varphi\right\rangle=\left\langle\pi\left(q_{j} x q_{j}\right) \varphi, \varphi\right\rangle \geqq 0 .
$$

Since $\pi$ is weakly continuous, $\langle\pi(x) \varphi, \varphi\rangle=\lim \left\langle\pi\left(q_{j} x q_{j}\right) \varphi, \varphi\right\rangle \geqq 0$. That is, $\pi(x) \geqq 0$ on $\mathscr{D}(\pi)$.

(ii): First let $x \in \mathscr{L}^{+}(\mathscr{D})_{h}$. Since $\pi$ is positive by (i) and $\pi(I)=I$, inf $\{\lambda \in \mathbb{R}:-\lambda I$ $\leqq \pi(x) \leqq \lambda I\} \leqq \inf \{\lambda \in R-\lambda I \leqq x \leqq \lambda I\}=\|x\|$, which implies that $\pi(x)$ is bounded and $\|\pi(x)\| \leqq\|x\|$. For arbitrary $x \in \mathscr{L}^{+}(\mathcal{D})$ the assertion follows from $\|\pi(x)\|^{2}=\left\|\pi\left(\dot{x}^{+} x\right)\right\|$, $\leqq\left\|x^{+} x\right\|=\|x\|^{2}$.

(iii): Suppose $x \in \mathscr{L}^{+}(\mathcal{D})$., By assumption; there are a positive constant $C$ and a natural number $s$ such that

Therefore,

$$
\|x \varphi\|^{2} \leqq C\left(\|\varphi\|^{2}+\sum_{n=1}^{s}\left\|x_{n}^{\prime} \varphi\right\|^{2}\right) \text { for all } \varphi \in \mathcal{D}
$$

The latter implies that

$$
y:=C\left(I+\sum_{n=1}^{\infty} x_{n}{ }^{+} x_{n}\right)-x^{+} x \in \mathscr{L}^{+}(\mathcal{D})_{+} \text {and } \pi(y) \geqq 0 \text { on } \mathscr{D}(\pi) \text {. }
$$

$$
\text { - }\|\pi(x) \varphi\|^{2} \leqq C\left(\|\varphi\|^{2}+\sum_{n=1}^{s} \cdot\left\|\pi\left(x_{n}\right) \varphi\right\|^{2}\right) \text { for all } \varphi \in \mathcal{D}(\pi)
$$

1.4 From now on we assume that $\mathcal{D}[t]$ is a Frechet space and that the underlying Hilbert space $\mathscr{H}$ is separable. To simplify the notation we adopt the following notational convention: We shall denote an operator whose domain contains $\mathscr{D}$ and its restriction to $\mathcal{D}$ by the same symbol. This will be mainly used in Section 3 . Let $\mathcal{F}(\mathscr{D})$ denote the finite rank operators contained in $\mathscr{L}^{+}(\mathscr{D})$. For a linear subspace $\mathscr{D}_{1}$ of $\mathscr{H}$, let $\mathscr{F}\left(\mathscr{H}, \mathscr{D}_{1}\right)$ be the set of all bounded finite-ranked operators on $\mathscr{H}$ mapping $\mathscr{H}$ into $\mathscr{D}_{1}$. Moreover, we let $\mathfrak{B}_{\mathscr{D}_{1}}:=\left\{\varphi \in \mathscr{D}_{1}:\|\varphi\| \leqq 1\right\}$.

\section{Generalized Calkin representations of $\mathcal{L}(D)$}

2.1 Suppose that $\mathcal{U}$ is an ultrafilter on $N$. Let $D_{\mathcal{U}}^{\infty}$ denote the set of all bounded sequences $\left(\varphi_{n}: n \in \mathrm{N}\right)=\left(\varphi_{n}\right)$ in the locally convex space $\mathcal{D}[l]$ satisfying $0=w$ - $\lim _{u} \varphi_{n}$. Let $\mathscr{H}_{u}^{\infty}$ be the set of all bounded sequences $\left(\varphi_{n}\right)$ in $\mathscr{H}$ with $0=w-\lim _{u} \varphi_{n} . \mathcal{D}_{u}^{\infty}$ and $\mathscr{H}_{u}^{\infty}$ are vector spaces in the obvious way. Let $\mathscr{N}_{\boldsymbol{u}}$ be the set of all $\left(\varphi_{n}\right) \in \mathscr{H}_{u}^{\infty}$ with $\lim _{\boldsymbol{u}}\left\|\varphi_{n}\right\|$ $=0$. We define a scalar product on the quotient space $\mathscr{D}_{u}:=\mathscr{D}_{u}^{\infty} / \mathscr{D}_{u}^{\infty} \cap \mathcal{N}_{u}$ by $\left\langle\left(\varphi_{n}\right)\right.$; $\left.\left(\psi_{n}\right)\right\rangle:=\lim _{u}\left\langle\varphi_{n}, \psi_{n}\right\rangle$. In the same way, the quotient space $\mathscr{H}_{u}:=\mathscr{H}_{u}^{\infty} \mid \mathcal{N}_{u}$ becomes a Hilbert space (see e.g. [11: Section 2]). By an abuse of notation we denote the elements of the quotient spaces again by $\left(\varphi_{n}\right)$. Since $\mathcal{D} \subseteq \mathscr{H}, \mathscr{D}_{\mathcal{u}}$ can be considered as a linear subspace of $\mathscr{H}_{u}$.

Define $\varrho u(x)\left(\varphi_{n}\right):=\left(x \varphi_{n}\right)$ for $\left(\varphi_{n}\right) \in \mathscr{D}_{u}$, and $x \in \mathscr{L}^{+}(\mathscr{D})$. Each operator $x \in \mathscr{L}^{+}(\mathscr{D})$ -maps a bounded sequence in $\mathscr{D}[t]$ into a bounded sequence. By Lemma 1.1, (i) and (ii), $x \mathscr{N}_{u} \subseteq \mathfrak{N}_{\mathcal{U}}$ and $x \mathscr{D}_{u}^{\infty} \subseteq \mathscr{D}_{u}^{\infty}$. Therefore, the above definition makes sense and defines a linear operator $\varrho u(x)$ which maps $\mathscr{D}_{u}$ into $\mathscr{D}_{u}$. It is straightforward to check that the mapping $x \rightarrow \underline{Q} u(x)$ is a positive *-representation of $\mathscr{L}^{+}(\mathscr{D})$ on $\mathscr{D}_{u}$.

Let $\mathrm{i}$ denote the quotient map of $\mathscr{L}^{\div}(\mathscr{D})$ onto $\mathcal{A}(\mathscr{D})=\mathscr{L}^{+}(\mathscr{D}) / \mathscr{C}(\mathcal{D})$. Suppose $x \in$ $\mathscr{C}(D)$ and $\left(\varphi_{n}\right) \in \mathscr{D}^{\infty}$. Then the set $\left\{x \varphi_{n}\right\}$ is relatively compact in $\mathscr{D}(l)$ and hence $\lim u\left\|x \varphi_{n}\right\|=0$ by Lemma 1.1 (iii). This, shows that $\mathscr{C}(\mathcal{D}) \leqq$ ker $\varrho u$. Therefore, $x_{u}(j(x))$ $:=\varrho_{u}(x)$ for $x \in \mathscr{L}^{+}(\mathscr{D})$ defines a *-representation of the *algebra $\mathcal{A}(\mathscr{D})$ on $D_{u} \equiv \mathcal{D}(\pi \hat{u})$. 
2.2 Recall that an ultrafilter on $N$ is said to be free if the intersection of all its members is empty.

Theorem 2.1: Suppose that $\mathcal{U}$ is a free ultrafilter on $N$. Then $\pi_{u}$ is a faithful.*representation of the Calkin algebra $\mathcal{A}(D)$. Its inverse $\pi u^{-1}$ is a continuous mapping of $\dot{\pi} u(\mathcal{A}(\mathcal{D}))\left[\tau_{\mathscr{D}_{u}}\right]$ onto $\mathcal{A}(\mathcal{D})[\hat{\imath}]$.

Proof: The quotient topology $\hat{\tau}$ on $\mathcal{A}(\mathscr{D})$ is generated by the seminorms

$$
\hat{p}_{\mathfrak{M}}(\mathrm{j}(x)):=\inf \left\{\operatorname{pom}_{\mathbb{R}}(x+c): c \in \mathscr{C}(\mathcal{D})\right\}, \mathfrak{M} \subset \mathscr{D}[t] \text { bounded. }
$$

Fix such a set $\mathfrak{M}$. Suppose for a moment we have shown that there, exists a bounded subset $\mathfrak{R}$ (depending on $\mathfrak{M}$ ) of $\mathcal{D}_{u}\left[t_{\mathrm{e} u}\right]$ such that

$$
\hat{p}_{\mathfrak{M}}(\mathrm{i}(x)) \leqq p_{\mathfrak{R}}(\varrho \varkappa(x)) \text { for all } x \in \mathscr{L}^{+}(\mathcal{D})
$$

The latter means that

$$
\hat{p}_{\mathfrak{M}}(a) \leqq p_{\mathfrak{R}}(\pi u(a)) \text { for all } a \in \mathcal{A}(\mathscr{D}) \text {. }
$$

Since $\mathscr{C}(\mathscr{D})$ is $\tau \mathscr{D}$-closed in $\mathscr{L}^{+}(\mathscr{D})$ and hence $\hat{\imath}$ is Hausdorff, it follows from (2) that ker $\pi u=\{0\}$, that is, $\pi u$ is faithful. Moreover, (2) proves the continuity of $\pi u^{-1}$, and the proof would be complete.

It remains' to show that there is a bounded set $\mathfrak{N}$ in $\left.\mathscr{D}_{u}\left[t_{\mathrm{Q}}\right]^{\prime}\right]$ such that (1) is satisfied. According to [6: Theorem 4.1] there is a bounded self-adjoint operator $z$ on $\mathscr{H}$ such that ker $z=\{0\}, z \mathscr{H} \subseteq \mathscr{D}$ and $\mathfrak{M} \subseteq z \mathfrak{B}_{\mathscr{H}}$. If $x \in \mathscr{L}^{+}(\mathscr{D})$, then $x z$ is a closed operator defined on $\mathscr{H}$ and hence bounded. Now fix an operator $x \in \mathscr{L}+(\mathscr{D})$. Since $\mathscr{F}(\mathscr{D})$ is

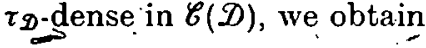

$$
\begin{aligned}
& \hat{p}_{\mathbb{M}}(\mathrm{i}(x)) \leqq \inf _{c \in \mathcal{F}(D)} p_{\mathfrak{M} R}(x+c) \\
& \cdots \cdot=\inf _{c \in \mathcal{F}(\mathscr{D})} \sup _{\varphi, \psi \in \mathcal{H}_{\mathscr{X}}}|\langle(x+c) z \varphi, z \psi\rangle|=\inf _{c \in \mathcal{I}(\mathscr{D})}\|z(x+c) z\| .
\end{aligned}
$$

Since $\operatorname{ker} z=\{0\}$, we have $\{c z: c \in \mathcal{F}(\mathcal{D})\}=\mathscr{F}(\mathcal{D})$. Moreover, $\{z c: c \in \mathcal{F}(\mathcal{D})\}$ is norm dense in $\mathscr{F}(\mathscr{H})$. Using these facts, we get

$$
\begin{aligned}
& \hat{p}_{\mathfrak{M}}(\mathrm{i}(x)) \leqq \inf _{c \in \mathcal{S}(\mathcal{D}) .}\|z x z+z c\| \\
& =\inf _{\epsilon \in \mathcal{S}(\mathscr{X})}\|z x z+c\|=\inf _{c \in \mathcal{C}(\mathscr{X})}\|z x z+\stackrel{\prime}{\prime}\| .
\end{aligned}
$$

On the other hand, let $\omega_{\mathcal{U}}$ denote the $*$-representation of $\mathrm{B}(\mathscr{H})$ on $\mathscr{H}_{\mathcal{U}}$ defined by $\omega_{u}(y)\left(\varphi_{n}\right):=\left(y \varphi_{n}\right)$. for $\left(\varphi_{n}\right) \in \mathscr{H}_{\boldsymbol{u}}$ and $y \in \mathrm{B}(\mathscr{H})$. Since $\omega_{\boldsymbol{u}}$ obviously annihilates, $\mathscr{C}(\mathscr{H}) ; \omega_{u}$ defines a $*$-representation of the Calkin algebra $\mathscr{A}(\mathscr{H})$ on $\mathscr{H}_{u}$ (see $[11$ : Section 2]). Since $\mathcal{U}$ is assumed to be free and $\mathcal{A}(\mathscr{H})$ is simple, this *-representation of the $C^{*}$-algebra $\mathcal{A}(\mathscr{H})$ is faithful and hence isometric. Since $z x z \in \mathrm{B}(\mathscr{H})$, this yields $\left\|\omega_{\varkappa}(z x z)\right\|=\inf \{\|z x z+c\|: c \in \mathscr{C}(\mathscr{H})\}$. By (3), we obtain

$$
\hat{p}_{\mathfrak{M}}(\mathrm{i}(x)) \leqq\left\|\omega_{u}(z x z)\right\| \text { for all } x \in \mathscr{L}^{+}(\mathcal{D}) \text {. }
$$

Now define

$$
\mathfrak{R}:=\omega_{\mathcal{U}}(z) \mathfrak{B}_{\mathfrak{H}_{u}} \equiv\left\{\left(z \varphi_{n}\right):\left(\varphi_{n}\right) \in \mathscr{H}_{\mathcal{U}} \text { and }\left\|\left(\varphi_{n}\right)\right\|_{\mathscr{H}_{u}} \leqq 1\right\} .
$$

If $\left(\varphi_{n}\right) \in \mathscr{H}_{u}$ and if $x \in \mathscr{L}^{+}(\mathscr{D})$, then $x z$ is bounded on $\mathscr{H}$ and thus

$$
\sup _{n \in \mathbb{N}}\left\|x z \varphi_{n}\right\| \leqq\|x z\| \sup _{n \in \mathbb{N}}\left\|\varphi_{n}\right\|<\infty .
$$


This implies $\mathfrak{N} \leqq \mathscr{D}_{\mathcal{U}}$. From

$$
\begin{aligned}
\left\|\varrho u(x)\left(z \varphi_{n}\right)\right\| & =\left\|\left(x z \varphi_{n}^{\prime}\right)\right\|_{\mathscr{H}_{u}}=\lim _{\boldsymbol{u}}\left\|x z \varphi_{n}\right\| \\
& \leqq\|x z\| \lim _{\boldsymbol{u}}\left\|\varphi_{n}\right\|=\|x z\| \text { for }\left(\varphi_{n}\right) \in \mathfrak{B}_{\mathscr{H}_{\mathcal{U}}} \text { and } x \in \mathscr{L}^{+}(\mathscr{D}),
\end{aligned}
$$

we see that $\mathfrak{R}$ is bounded in $\mathscr{D}_{u}\left[t_{e} u\right]$.

Finally, by (4), if $x \in \mathscr{L}^{+}(\mathscr{D})$, then

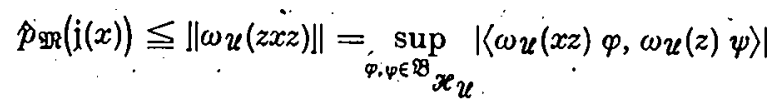

$$
\begin{aligned}
& =\sup _{\left(\varphi_{n}\right),\left(\varphi_{n}\right) \in \mathfrak{B}_{\mathscr{B}_{\mathcal{U}}}}\left|\left\langle\left(x z \varphi_{n}\right),\left(z \psi_{n}\right)\right\rangle\right|
\end{aligned}
$$

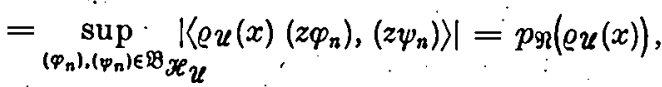

which proves (1). The proof of Theorem 2.1 is complete

\subsection{From Theorem 2.1 and Lemma 1.4 we obtain}

Theorem 2.2: Suppose that $\tau_{n}=\tau_{\mathscr{D}}$ on $\mathscr{L}^{+}(D)$. Let $\mathcal{U}$ be a free ultrafilter, on $\mathrm{N}$. Then, $\pi_{u}$ is a faithful *-representation of $\mathcal{A}(\mathcal{D})$ and a homeomorphism of $\mathcal{A}(\mathscr{D})[\hat{\tau}]$ onto $\pi_{u}(\mathcal{A}(D))\left[\tau_{\mathscr{D}} \boldsymbol{u}\right]$.

1. In general the domain $\mathscr{D}_{u}$ is not dense in. $\mathscr{H}_{u}$. 2. If the domain is of the form $\mathscr{D}=n\left\{\mathscr{D}\left(T^{n}\right): n \in \mathrm{N}\right\}$ for some self-adjoint operator $T$ on $\mathscr{H}$, then $\tau_{n}=\tau_{\mathscr{D}}$ on $\mathscr{L}+(\mathscr{D})$ (see also Section 3).

\section{Existence of continuous faithful $\bullet$-representations. of $\mathscr{A}(\mathcal{D})[\hat{\imath}]$}

3.1 We first recall the setup of [14: Section 4]. However, the notation is slightly changed.

Suppose $a$ is a (bounded or unbounded) self-adjoint operator on the Hilbert space $\mathscr{H}$ with spectral decomposition $a=\int \lambda \cdot d e(\lambda)$. Let $\left(f_{k}(t): k \in N\right)$ be a sequence of real measurable functions on the spectrum $\sigma(a)$ of $a$. All measure-theoretic notions refer to the spectral measure of $a$. We assume that

$$
f_{1}(t)=1 \text { and } f_{k}(t) \leqq f_{k}{ }^{2}(t) \leqq f_{k+1}(t) \quad \text { a.e. on } \sigma(a) \text { for } k \in N .
$$

Set $a_{k}=f_{k}(a)$ and $\mathcal{D}=n\left\{\mathcal{D}\left(a_{k}\right): k \in N\right\}$. Then, by $(1)$, the operators $a_{k}$ (more precisely, their restrictions to $\mathscr{D})$ are in $\mathscr{L}^{+}(\mathscr{D})$ and the graph topology $t$ on $\mathcal{D}$ is generated by the seminorms $\|\cdot\|_{a_{k}}, k \in N$.

In our next theorem the following condition (*) plays an important role:

(*) $\left\{\begin{array}{l}\text { For each sequence } \gamma=\left(\gamma_{k}: k \in N\right) \text { of positive numbers } \gamma_{k} \text { there is a } \\ k=k_{y} \in N \text { such that all functions } f_{n}, n \in N \text {, are bounded on } \mathfrak{R}_{k}, \text { where } \\ \mathfrak{R}_{n}:=\left\{t \in \sigma(a): f_{1}(t) \leqq \gamma_{1}, \ldots, f_{n}(t) \leqq \gamma_{n}\right\} \text { for } n \in \mathrm{N} .\end{array}\right.$

The following assertions are equivalent:

(i) Condition (*) is fulfilled.

(ii) , $\tau_{0}=\tau_{\mathscr{D} \mid}$ on $\mathscr{L} \div(\mathscr{D})$.

(iii) $\tau_{n}=\tau_{\mathscr{D}}$ on $\mathscr{L}^{+}(\mathscr{D})$.

(iv) Each positive linear functional on $\mathscr{L}^{+}(D)$ is $\tau_{\mathscr{D}}$-continuous. 
This is essentially [14: Theorem 4:1]. The equivalence of (i), (ii) and (iv) has been stated therein. Since $\tau_{0} \supseteqq \tau_{n} \supseteqq \tau_{\mathscr{D}}$, (ii) $\Rightarrow$ (iii). Since each positive linear functional is $\tau_{n}$-continuous, we have (iii) $\Rightarrow$ (iv).

3.2 The following theorem may be considered as a supplement to [14: Theorem 4.1]. Among other things it shows that if $\tau_{n} \neq \tau_{\mathscr{D}}$ on $\mathscr{L}^{+}(\mathscr{D})$, then there is no continuous faithful *-representation of $\mathcal{A}(\mathscr{D})[\hat{\tau}]$. In particular, the *-representations $\pi \varkappa$ occuring in Theorem 2.1 are not continuous.

Theorem 3.1: Let $\mathcal{D}$ be as above. Then (i) is equivalent to each of the following conditions:

(v) There exists a faithful *-representation $\pi$ of $\mathcal{A}(\mathcal{D})$ which is a homeomorphism of $\mathcal{A}(\mathcal{D})[\hat{\imath}]$ onto $\pi(\mathcal{A}(\mathscr{D}))\left[\tau_{\mathscr{D}(\pi)}\right]$.

(v) There exists a continuous faithful *-representation of $\mathscr{A}(\mathscr{D})[\hat{\imath}]$.

(vi) Each positive *-representation of $\mathscr{L}^{+}(\mathscr{D})\left[\tau_{\mathscr{D}}\right]$ is continuous.

(vi)' Each weakly continuous positive *-representation of $\mathscr{L}^{+}(\mathcal{D})\left[\tau_{\mathscr{D}}\right]$ is continuous.

Proof: Theorem 2.2 shows that (iii) $\Rightarrow(\nabla)$. (iii) $\Rightarrow$ (vi) follows from Lemma 1.3. Since $(v) \Rightarrow(v)^{\prime}$ and $(v i) \Rightarrow(v i)^{\prime}$ are trivially fulfilled, it suffices to prove that $(\nabla)^{\prime}$ $\Rightarrow(i)$ and $(v i)^{\prime} \Rightarrow$ (i). Both proofs will be indirect (see e.g. the argument in [14: p. 366]).

$(v)^{\prime} \Rightarrow$ (i) : Suppose that $\pi$ is a continuous faithful $*$-representation of $\mathcal{A}(\mathscr{D})[\hat{\tau}]$. Then, $\varrho(x):=\pi(\mathrm{i}(x)), x \in \mathscr{L}^{+}(\mathscr{D})$, defines a continuous $*$-representation of $\mathscr{L}^{+}(\mathcal{D})\left[\tau_{\mathbb{D}}\right]$. To prove (i), we assume the contrary, that is', condition. (*) is not satisfied. Then there are a positive sequence $\gamma=\left(\dot{\gamma_{k}}\right)$ and a sequence $\left(i_{k}\right)$ of natural numbers such that $f_{i_{k}}$ is not essentially bounded on the set $\mathfrak{\Re}_{k}$ for each $k \in N$. There is no loss of generality if we assume that $\gamma_{k+1}>\gamma_{k} \geqq k$ and $i_{k}=k$ for all $k \in N$. Then there are measurable subsets $\Im_{k, n}, n \in N$, of $\mathfrak{R}_{k}$ of non-zero measure such that $f_{k+1}(t) \geqq \gamma_{n}$ a.e. on $\Im_{k, n}$ for all $k, n \in \mathbb{N}$. Let $\varphi_{k, n}$ be a unit vector from $e\left(\Im_{k, n}\right) . \mathscr{D}$.

Let $\Delta$ denote the family of all sequences $\delta=\left(\delta_{k}\right)$ of natural numbers $\delta_{k}$ satisfying $\delta_{k} \geqq k+2$ for $k \in N$. Fix a $\delta \in \Delta$. We first show that for $r \in N$ and $\varphi \in \mathscr{D}(\varrho)$

$$
\left\|\varrho\left(a_{r}\right) \varrho\left(e\left(\bigcup_{k \geqq r+1} \Im_{k, \delta_{k}}\right)\right) \varphi\right\| \leqq \gamma_{r}\|\varphi\|
$$

and

$$
\left\|\varrho\left(a_{r+1}\right) \varrho\left(e\left(\Im_{r, \delta_{r}}\right)\right) \varphi\right\| \geqq \gamma_{\delta_{r}}\left\|\varrho\left(e\left(\Im_{r, \delta_{r}}\right)\right) \varphi\right\| .
$$

For let- $\chi$ denote the characteristic function of the set $\cup\left\{\Im_{k, \delta_{k}}: k \geqq r+1\right\}$. By construction, $f_{r}(t) \chi(t) \leqq \gamma_{r}$ a.e. on $\dot{\sigma}(a)$. Define a function $g$ on $\sigma(a)$ by $g:=\left(\gamma_{r}^{2}-f_{r}^{2} \chi\right)^{1 / 2}$. Obviously, $g(a) \in \mathscr{L}^{+}(\mathcal{D})$. For $\varphi \in \mathscr{D}(\varrho),\left\langle\varrho\left(g(a)^{2}\right) \varphi, \varphi\right\rangle=\|\varrho(g(a)) \varphi\|^{2} \geqq 0$ and hence

$$
\begin{aligned}
&\|\varphi\|^{2} \gamma_{r}^{2}=\left\langle\varrho\left(\gamma_{r}^{2} I\right) \varphi, \varphi\right\rangle \\
& \quad \geqq\left\langle\varrho\left(f_{r}(a)^{2} \chi(a)\right) \varphi, \varphi\right\rangle=\left\|\varrho\left(a_{r}\right) \varrho\left(e\left(\underset{k \geqq r+1}{\cup} \Im_{k, \delta_{k}}\right)\right) \grave{\varphi}\right\|^{2}:
\end{aligned}
$$

(2) follows by the same argument.

Let $q_{\delta}$ be the orthogonal projection onto the closure of $D_{\delta}:=1 . h .\left\{\varphi_{k . \delta_{k}}: k \in N\right\}$. Next we prove that $q_{\delta} \mathscr{H} \subseteq \mathscr{D}$. For let $r \in N$. Each $\varphi \in \mathscr{D}_{\delta}$ can be written as a finite sum

$$
\sum_{k=1}^{s} \lambda_{k} \varphi_{k, \delta_{k}}, \text { where } \lambda_{1}, \ldots, \lambda_{s} \in C \text { and } s \in N, s>r .
$$

Suppose $k, n \in \dot{N}, n>k$. Since $f_{k+1}(t) \geqq \gamma_{\delta_{k}} \geqq \gamma_{k+2}>\gamma_{k+1}$ a.e. on $\Im_{k . \delta_{k}}$ and $f_{k+1}(t)$ $\leqq \gamma_{k+1}$ on $\Im_{n, \delta_{n}}$, it follows that $\Im_{k, \delta_{k}} \cap \Im_{n, \delta_{n}}$ has measure zero. Therefore, $\varphi_{k, \delta_{k}}$ 
$\perp \varphi_{n, \delta_{n}}$ and $a_{r} \varphi_{k, \delta_{k}} \perp a_{r} \dot{\varphi}_{n, \delta_{n}}$. Using the latter, we obtain

$$
\begin{aligned}
\left\|a_{r} \varphi\right\|^{2} & =\sum_{k=1}^{r}\left|\lambda_{i_{k}}\right|^{2}\left\|a_{r} \varphi_{k, \delta_{k}}\right\|^{2}+\sum_{k=r+1}^{s}\left|\hat{\lambda}_{k}\right|^{2}\left\|a_{r} \varphi_{k, \delta_{k}}\right\|^{2} \\
1 . & \leqq \max \left(\left\|a_{r} \varphi_{1, \delta_{2}}\right\|, \ldots,\left\|a_{r} \varphi_{r, \delta_{r}}\right\|, \gamma_{r}\right) \sum_{k=1}^{s}\left|\dot{\lambda}_{k}\right|^{2}=\max (\ldots)\|\varphi\|^{2} .
\end{aligned}
$$

This implies $q_{e} \mathscr{H}, \subseteq \mathscr{D}\left(a_{r}\right)$. Since $\mathscr{D}=\cap\left\{\mathscr{D}\left(a_{r}\right): r \in \mathrm{N}\right\}$ by definition, this shows that $q_{\rho} \mathscr{H} \subseteq \mathscr{D}$.

We define $\mathfrak{A}:=\bigcup\left\{\varrho\left(q_{\delta}\right) \mathfrak{B}: \delta \in \Delta\right\}$, where $\mathfrak{B}:=\mathfrak{B}_{\mathcal{D}_{(\varrho)}} \equiv\{\varphi \in \mathscr{D}(\varrho):\|\varphi\| \leqq 1\}$. We prove that $\Re$ is bounded in $\mathscr{D}(\varrho)$ [ $\left.t_{e}\right]$. For take $r \in \mathfrak{N}$ and $\delta \in \Delta$. Let $c_{r, \delta}$ denote the orthogonal projection on $\mathscr{H}$ with range l.h. $\left\{\varphi_{1, \delta_{1}}, \ldots, \varphi_{r, \delta_{r}}\right\}$. Since obviously $a_{r} c_{r, \delta}$ $\in \mathscr{C}(D)$, we have $a_{r} c_{r, \delta} \in$ ker $\varrho$. From

$$
q_{\delta}-c_{r, \delta}=e\left(\bigcup_{k \geq r+1} \widetilde{\Im}_{k, s_{k}}\right) \cdot\left(q_{\delta}-e_{r, \delta}\right)
$$

and (2) we therefore obtain

$$
\begin{aligned}
\left\|\varrho\left(a_{r}\right) \varrho\left(q_{\delta}\right) \varphi\right\| & =\left\|\varrho\left(a_{r}\right) \varrho\left(q_{\delta}-c_{r, \delta}\right) \varphi\right\| \\
& =\left\|\varrho\left(a_{r}\right) \varrho\left(e\left(\underset{k \geqq r+1}{\cup} \Im_{k, \delta_{k}}\right)\right) \varrho\left(q_{\delta}-c_{r, \delta}\right) \varphi\right\| \\
& \leqq \gamma_{r}\left\|\varrho\left(q_{\delta}-c_{r, \delta}\right) \varphi\right\| \leqq \gamma_{r} \text { for each } \varphi \in \mathfrak{B}
\end{aligned}
$$

By Lemma 1.4 (iii) the graph topology $t_{\rho}$ on $\mathscr{D}(\varrho)$ is generated by the seminorms $\|\cdot\|_{e\left(a_{r}\right)}, r \in N$. Therefore, the preceding proof-shows that $\mathfrak{N}$ is bounded with respect to the graph topology $t_{e}$.

Since the *-representation $\varrho$ of $\mathscr{L}^{+}(\mathscr{D})\left[\tau_{\mathscr{D}}\right]$ is continuous, there exists a bounded subset $\mathfrak{M}$ of $\mathcal{D}(t)$ such that

$$
p_{\mathfrak{M}}(\varrho(x)) \leqq p_{\mathfrak{M}}(x) \text { for all } x \in \mathscr{L}^{+}(\mathscr{D})
$$

Since $\mathfrak{M}$ is $i$-bounded, $\left.C_{r}:=\sup \left\{\left\|\varrho\left(a_{r}\right) \varphi\right\|: \varphi \in \mathfrak{M}\right\}\right\}<\infty$ for each $r \in N$. We choose natural numbers $\delta_{k}$ such that $\delta_{k} \geqq k+2$ and $\gamma_{\delta_{k}} \geqq C_{k+1} 2^{k}$ for $k \in N$. This is possible because $\gamma_{n} \geqq n$ for $n \in N$. Define an operator $x$ by $x:=e\left(\cup\left\{\Im_{k, \delta_{k}}: k \in N\right\}\right)$. Clearly, $x \in \mathscr{L}^{+}(\mathscr{D})$ : Our aim is to show that for this operator $x(4)$ is not true. By (3), we have

That is;

$$
\begin{aligned}
\gamma_{\delta_{r}}\left\|\varrho\left(e\left(\Im_{r, \delta_{r}}\right)\right) \varphi\right\| & \leqq\left\|\varrho\left(a_{r+1}\right) \varrho\left(e\left(\Im_{r, \delta_{r}}\right)\right) \varphi\right\| \\
& \leqq\left\|\varrho\left(a_{r+1}\right) \varphi\right\| \leqq C_{r+1} \text { for }-r \in N \text { and } \varphi \in M_{\ell} .
\end{aligned}
$$

$$
\sup _{\varphi \in \mathbb{W} t}\left\|\varrho\left(e\left(\widetilde{\mho}_{r, \delta_{r}}\right)\right) \varphi\right\| \leqq C_{r+1} \gamma_{\delta_{r}}^{-1} . \text { for } \quad r \in \mathbb{N} .
$$

Using this inequality, we obtain .

$$
\begin{aligned}
p_{\mathbb{M}}(x) & =\sup _{\varphi, \varphi \in \mathfrak{M} R}\left|\left\langle e\left(\bigcup_{k} \Im_{k, \delta_{k}}\right) \varphi, \psi\right\rangle\right| \\
& \leqq \sup _{\rho \in \mathfrak{P}} \sum_{k=1}^{\infty}\left\|e\left(\Im_{k, \delta_{k}}\right) \varphi\right\|^{2} \leqq \sum_{k=1}^{\infty} C_{k ! 1}^{2} \gamma_{\delta_{k}}^{-2} \leqq \sum_{k=1}^{\infty} 2^{-2 k}<1
\end{aligned}
$$

Since $a_{r} q_{\delta}$ is a-bounded operator on $\mathscr{H}$ for $r \in N$ as shown above, the sequence $\left(\varphi_{k, \delta_{k}}: k \in N\right)$ is bounded in $\mathscr{D}(t)$. But the set $\left\{q_{\delta} \varphi_{k, \delta_{k}}\right\}=\left\{\varphi_{k, \delta_{k}}\right\}$ is certainly not relatively compact-in $\mathscr{D}[l]$, since $\left(\varphi_{k, \delta_{k}}\right)$ is an orthonormal sequence in. $\mathscr{H}$. This proves that 
$q_{\delta} \notin \mathscr{C}(\mathscr{D})=$ ker $\varrho$. Consequently,

$$
\begin{aligned}
1 & =\sup _{\varphi \in \mathfrak{B}}\left\|\varrho\left(q_{\delta}\right) \varphi\right\|^{2} \\
& =\sup _{\varphi \in \mathfrak{B}}\left|\left\langle\varrho\left(e\left(\bigcup_{k} \Im_{k, \delta_{k}}\right)\right) \varrho\left(\grave{q}_{\delta}\right) \varphi, \varrho\left(q_{\delta}\right) \varphi\right\rangle\right|_{\downarrow} \leqq p_{\mathfrak{R}}(\varrho(x)) .
\end{aligned}
$$

Comparing (5) and (6) with (4), we obtain the desired contradiction.

$(v i)^{\prime} \rightarrow$ (i) : This will be similar as the preceding proof. Again we assume that condition (*) is not fulfilled. We keep the notation introduced above. Let $u$ be an arbitrary free ultrafilter on N. As already mentioned in Section 2, $\varrho u$ is a positive *-representation of $\mathscr{L}^{+}(D)$. It suffices to show that $\varrho u^{-}$is weakly continuous, but not continuous. Let $\varphi=\left(\varphi_{n}\right) \in \mathcal{D}_{\mathcal{u}}$. By definition of $\mathcal{D}_{\mathcal{u}}$, the set $\mathfrak{R}:=\left\{\varphi_{n}\right\}$ is bounded in $\mathscr{D}[t]:$ If $x \in \mathscr{L}^{+}(\mathscr{D})$, then

$$
|\langle\varrho u(x) \varphi, \varphi\rangle|=\left|\lim _{u}\left\langle x \varphi_{n}, \varphi_{n}\right\rangle\right| \leqq \sup _{n \in \mathbb{N}}\left|\left\langle x \varphi_{n}, \varphi_{n}\right\rangle\right| \leqq p_{\mathbb{M}}(x) .
$$

That is, $\varrho u$ is weakly continuous'. From Theorem 2.1 we know that ker $\varrho u=\mathscr{C}(D)$. Therefore, the preceding proof in the case $\varrho=\varrho u$ shows that $\varrho \dot{u}$ is not $\tau_{D}$-continuous [14]).

Addendum: After completing the manuscript the author has learned that in the case $\mathscr{D}=n\left\{D\left(T^{n}\right): n \in \mathrm{N}\right\}, T$ a self-adjoint operator, the existence of a topological realization of $\mathscr{A}(\mathscr{D})[\hat{\imath}]$ has been independently obtained by F. LöFFLER and W. TMMrERMa representation for a certain class of algebras of unbounded operators", Dubna-Preprint E 5-84-807, 1984.

\section{REFERENCES}

[1] ARnaL, D., and J. P. JURZAK: Topological aspects of algebras of unibounded operators J. Funct. Anal. 24 (1977), $397-425$.

[2] Bodrbaki, N.: Topologie générale. Paris: Hermann 1961.

[3] CaLkriv, J. W.: Two-sided ideals and congruences in the ring of bounded operators in Hilbert space. Ann. Math. 42 (1941), $839-873$ :

[4] Hennrich, S.: Ultrapowers of locally convex spaces and applications. Preprint. Berlin: Akad. Wiss. DDR, Preprints 10, 11 (1983).

[5] JunEK, H. J.: Locally Convex Spaces and Operators Ideals (Teubner-Texte zur Mathematik: Vol. 56). Leipzig: BSB B. G. Teubner Verlagsgesellschaft 1983.

[6] Kürstev, K.-D.: The completion of the maximal Op*-algebra on a Frechet domain. Publ. RIMS Kyoto Univ. 22 (1986), $151-175$.

[7] KürSTEN, K.-D.: Two-sided ideals of certain algebras of unbounded operators. To appear.

[8] Lasswer, G.: Topological algebras of operators. Reports Math. Phys. 3 (1972), 279-293.

[9] ЛёфФлЕР, Ф.: Свойства алгебр неограниченных операторов заданных на плотном множестве и замкнутые идеалы в иих. Дипломная работа. Харьков: Государствениый университет 1983 .

[10] Powers, R. T.: Self-adjoint algebras of unbounded operators. Commun. Math. Phys. 21 (1971), 85-124.

[11] ReId, G. A.: On the Calkin representations. Proc. London Math. Soc. 23 (1971), 547 - 564.

[12] SchÄfer, H. H.: Topological vector spaces. New York: Mac Millan Comp. 1966. 
[13] Schmübaen, K.: The order structure of topological *-algebras of unbounded operators. Report Math. Phys. 7 (1975), 215-227.

[14] SormüdGEN, K.: On topologization of unbounded operator algebras. Reports Math. Phys. 17 (1980), 359-371.

[15] Trmmermans, W.: Simple properties of some ideals of compact operators in algebras of unbounded operators. Math. Nachŕ. 90 (1979), 189-196.

Manuskripteingang: 16. 01: 1985

VERFASSER:

Prof.' Dr. Konrad Schmüdgen

Sektion Mathematik der Karl-Marx-Universität

DDR - 7010 Leipzig, Karl-Marx-Platz 